\title{
USO DE CHUPETA E SUA RELAÇÃO COM ALEITAMENTO MATERNO PROLONGADO NA CIDADE DE FEIRA DE SANTANA/BAHIA
}

\section{Romário Carneiro de Oliveira'; ${ }^{1}$ Abdias Medeiros de oliveira²; Suelly Pinto Teixeira de Morais ${ }^{3}$; Vinicius Carneiro de Oliveira ${ }^{4}$}

1 Bolsista FAPESB, Graduando em Medicina, Universidade Estadual de Feira de Santana, romariocarneiro.5@hotmail.com.

2 Orientador, Departamento de Saúde, Universidade Estadual de Feira de Santana, Abdias@uefs.br.

3 Participante do NUPES, Departamento de Saúde, Universidade Federal do Recôncavo Baiano, Santo Antonio de Jesus, suelly.pinto@terra.com.br.

4. Bolsista PIBD, Graduando em Medicina, Universidade Estadual de Feira de Santana, viniciuscarneiro.5@hotmail.com.

PALAVRAS-CHAVE: Amamentação prolongada; chupeta; aleitamento materno.

\section{INTRODUÇÃO:}

A alimentação é de grande importância para o desenvolvimento físico e psicológico da criança, desde o momento de seu nascimento ${ }^{1,2}$. A World Health Organization $(\mathrm{WHO})^{3}$ recomenda que as crianças sejam amamentadas exclusivamente até os seis meses de vida e a partir desta idade o aleitamento materno deve ser associado a introdução de alimentos complementares e manter o aleitamento materno prolongado até pelo menos os 2 anos de idade.

$\mathrm{O}$ uso de bicos e chupetas está constantemente associado às práticas de desmame, numa magnitude tão expressiva que entre os dez passos para o sucesso do aleitamento materno, o nono passo é a orientação de não oferecer bicos ou chupetas às crianças amamentadas ao seio ${ }^{3}$. Na literatura existem várias explicações para esta relação, a chupeta é considerada como um marcador de dificuldade na amamentação e, também está associada a uma confusão de bicos e redução do número de mamadas por dia, levando a menor produção de leite ${ }^{4,5}$.

$\mathrm{O}$ uso de chupeta também interfere no aleitamento materno prolongado, no estudo de NETO, ZANDONADE e EMMERICH (2013) ${ }^{7}$ a sucção de chupeta aumentou em 3,4 vezes a chance de desmame em criança menor que 1 ano de vida. MARTINS e GIUGLIANI (2012) $)^{8}$ também acharam esta relação, a manutenção do AM por 2 anos ou mais foi 2,4 vezes mais freqüente entre mães que filhos não tiveram o hábito de usar chupeta.

O atual estudo tem como objetivo principal entender os fatores determinantes da relação entre o uso de chupetas e o aleitamento materno prolongado.

\section{MATERIAL E MÉTODO:}

A análise dos dados foi realizada em três etapas. Na primeira etapa informações sobre as variáveis foram descritas em valor absoluto e relativo (prevalência). Na segunda etapa, foram calculadas as medidas de associação (Razão de Prevalência) e de significância estatística (Qui-Quadrado) adotando-se nível de 5\% de significância estatística, apenas para a variável preditora principal (uso de chupeta) e a variável desfecho (interrupção do AMP) nos períodos de 6, 9, 12, 18 e 24 meses de vida da criança. 
Na terceira etapa, utilizou-se o modelo de regressão logística (RL) para avaliar o efeito dos fatores de interesse sobre a probabilidade de interromper o AMP no $24^{\circ}$ mês de vida da criança. Inicialmente, as variáveis de interesse foram incluídas individualmente no modelo (oddsratiobruta - OR bruta). Na próxima etapa foi construído um modelo inicial de RL, seguindo o método backward, onde todas as variáveis foram ajustadas (OR ajustada), permanecendo no modelo final apenas as variáveis que obtiveram $p<0,20$. No modelo final de $\mathrm{RL}$ foram estimados os coeficientes de regressão, os valores da OR e seus intervalos de confiança de $95 \%$ (IC95\%), estipulando o valor $p \leq 0,05$ para significância estatística. O modelo final foi avaliado por meio do teste de bondade de ajuste de Hosmer-Lemeshow $(p>0,05)$.

Para realizar a etapa descritiva da análise estatística, utilizamos o programa StatisticalPackage for the Social Sciences (SPSS) versão 20.0. Para as etapas da fase analítica foi utilizado o programa Stata versão 12.0.

\section{RESULTADOS:}

No início da coorte 1309 indivíduos aceitaram participar do estudo e integraram a análise descritiva da amostra do estudo. Ao longo da coorte houve perda de seguimento de alguns desses participantes totalizando 908 indivíduos no final do estudo, ao $24^{\circ}$ mês de acompanhamento. O tamanho da amostra por período analisado está apresentado na Tabela 2.

As características maternas e infantis da amostra de 1309 indivíduos, são descritas na Tabela 1. Teve maior prevalência de mães primíparas, com 20 anos ou mais e que trabalhavam em casa, e de crianças que nasceram a termo com peso normal.

A tendência do uso de chupeta foi diminuir de acordo com a idade da criança, o índice diminuiu de $51,0 \%$ no sexto mês para $33,0 \%$ no $24^{\circ}$ mês. Com relação a interrupção do AMP observou-se aumento da prevalência segundo a idade da criança, de $26,0 \%$ no sexto mês aumentou para $79,2 \%$ no $24^{\circ}$ mês.

O uso de chupeta apresentou associação positiva para a interrupção do AMP, em todos os períodos analisados $(p=0,00)$, sendo maior a prevalência de interrupção do AMP entre as crianças que usavam chupeta no $24^{\circ}$ mês de vida ( $R P=7,62$; IC95\%: 4,14-14,02) (Tabela 3).

No modelo inicial, observou-se que duas das dez variáveis avaliadas não apresentaram associação com a interrupção do AMP, com valor $p<0,20$, sendo estas: tipo de parto e trabalho materno fora do lar. Entre as oito variáveis analisadas, apenas três mantiveram associação estatisticamente significativa $(p \leq 0,05)$ e permaneceram no modelo final.

As mães que introduziram chupeta no $24^{\circ}$ mês de vida da criança tiveram 12 vezes mais chance de interromper o AMP (IC95\%:6,49-24,18). Já para as mães primíparas a chance de interrupção do AMP no $24^{\circ}$ mês de vida da criança foi de $50 \%$ (IC95\%: 1,06-2,13) comparadas as multíparas. A escolaridade materna associou-se negativamente a interrupção do AMP, as mães que estudaram até o ensino fundamental tiveram $55 \%$ de chance a mais de continuar o AMP no $24^{\circ}$ mês de vida da criança (IC95\%: 0,39-0,78) do que aquelas com maior escolaridade. 
Tabela 3.Associação entre uso de chupeta e interrupção do aleitamento materno prolongado durante os períodos analisados.

\begin{tabular}{|c|c|c|c|c|c|c|c|c|}
\hline \multirow{3}{*}{$\begin{array}{c}\text { Uso de } \\
\text { chupet } \\
\mathbf{a}\end{array}$} & & \multirow{3}{*}{$\mathbf{N}$} & \multicolumn{4}{|c|}{$\begin{array}{l}\text { Interrupção do aleitamento } \\
\text { materno prolongado }\end{array}$} & \multirow{3}{*}{ RP $(\mathrm{IC} 95 \%) *$} & \multirow{3}{*}{ p-valor } \\
\hline & & & \multicolumn{2}{|c|}{ Sim } & \multicolumn{2}{|c|}{ Não } & & \\
\hline & & & $\mathbf{n}$ & $\%$ & $\mathbf{n}$ & $\%$ & & \\
\hline \multirow{2}{*}{$6^{0}$ mês } & Sim & \multirow{2}{*}{1134} & 247 & $42,7 \%$ & 331 & $57,3 \%$ & $\begin{array}{c}2,12 \\
(1,92-2,34)\end{array}$ & 0,000 \\
\hline & Não & & 48 & $8,6 \%$ & 508 & $91,4 \%$ & & \\
\hline \multirow{2}{*}{$9^{\circ}$ mês } & Sim & \multirow{2}{*}{1074} & 325 & $64,7 \%$ & 117 & $35,3 \%$ & $\begin{array}{c}2,77 \\
(2,42-3,18)\end{array}$ & 0,000 \\
\hline & Não & & 103 & $18,0 \%$ & 469 & $82,0 \%$ & & \\
\hline \multirow{2}{*}{$12^{\circ}$ mês } & Sim & \multirow{2}{*}{1037} & 332 & $74,3 \%$ & 115 & $25,7 \%$ & $\begin{array}{c}3,06 \\
(2,58-3,64)\end{array}$ & 0,000 \\
\hline & Não & & 171 & $29,0 \%$ & 419 & $71,0 \%$ & & \\
\hline \multirow{2}{*}{$18^{\circ}$ mês } & Sim & \multirow{2}{*}{984} & 344 & $89,1 \%$ & 42 & $10,9 \%$ & $\begin{array}{c}4,50 \\
(3,36-6,03)\end{array}$ & 0,000 \\
\hline & Não & & 291 & $48,7 \%$ & 307 & $51,3 \%$ & & \\
\hline \multirow{2}{*}{$24^{\circ}$ mês } & Sim & \multirow{2}{*}{908} & 290 & $96,7 \%$ & 10 & $3,3 \%$ & $\begin{array}{c}7,62 \\
(4,14-14,02)\end{array}$ & 0,000 \\
\hline & Não & & 429 & $70,6 \%$ & 179 & $29,4 \%$ & & \\
\hline
\end{tabular}

*RP: razão de prevalência. IC95\%: intervalo de 95\% confiança

\section{CONSIDERAÇÕES FINAIS:}

Os resultados encontrados reforçam a necessidade de se continuar estimulando o aleitamento materno exclusivo, a importância de informar as mães sobre as desvantagens do uso da chupeta e da implementação de políticas públicas que protejam o binômio mãe/criança.

\section{REFERÊNCIAS:}

1. Diehl, J. P; Anton, M. C. 2011. Fatores emocionais associados ao aleitamento materno exclusivo e sua interrupção precoce: um estudo qualitativo. Aletheia. (34):4760.

2. Victora, C. G; Aluísio, J. D; Barros, A. J. D; França, G. V. A. et al. 2016. Breastfeeding in the 21st century: Epidemiology, mechanisms, and lifelong effect. Lancet. 387(10017): 475-490.3.

3. World Health Organization. The optimal duration of exclusive breastfeeding. Report of an Expert Consultation - Geneva, Switzerland, 2001. 
4.Victora, C. G; Behague, D. P; Barros, F. C; Olinto, M. T; Weiderpass, E. 1997. Pacifier use and short breastfeeding duration: cause, consequence, or coincidence? Pediatrics. 99(3):445-53.

5.Kramer, M. S; Barr, R. G; Dagenais, S; Yang, H; Jones, P; Ciofani, L. et al. 2001. Pacifier use, early weaning, and cry/fuss behavior: a randomized controlled trial. Jama. 286(3):322-6.

6. Lopes, P. T. S; Moura, L. F. A. D; Lima, M. C. M. P. 2014. Breastfeeding and sucking habits in children enrolled in a mother-child health program. BMC Research Notes. 7:362.

7. Gaíva, M. A. M; Medeiros, L. S. 2006. Lactação insuficiente: uma proposta de atuação do enfermeiro. Ciênc. cuid. saúde. 5(2): 255-62. 